\title{
DECOMPOSITIONS OF VECTOR MEASURES IN RIESZ SPACES AND BANACH LATTICES
}

\author{
by KLAUS D. SCHMIDT
}

(Received 30th October 1984)

\section{Introduction}

The present paper is mainly concerned with decomposition theorems of the Jordan, Yosida-Hewitt, and Lebesgue type for vector measures of bounded variation in a Banach lattice having property $(P)$. The central result is the Jordan decomposition theorem due to which these vector measures may alternately be regarded as order bounded vector measures in an order complete Riesz space or as vector measures of bounded variation in a Banach space. For both classes of vector measures, properties like countable additivity, purely finite additivity, absolute continuity, and singularity can be defined in a natural way and lead to decomposition theorems of the Yosida-Hewitt and Lebesgue type. In the Banach lattice case, these lattice theoretical and topological decomposition theorems can be compared and combined.

This paper is organized as follows:

In Section 2 we consider order bounded vector measures in an order complete Riesz space. We first note that the class of all order bounded vector measures is an order complete Riesz space itself. From this fact, the Jordan decomposition theorem of Faires and Morrison [5] is immediate, and further decomposition theorems can be deduced by pure Riesz space techniques. We thus obtain the Yosida-Hewitt band decomposition theorem of Congost Iglesias [2] and a new Lebesgue band decomposition theorem. We also generalize the Lebesgue null-set decomposition theorem of Pavlakos [8] and Congost Iglesias [2] for vector measures in a super Dedekind complete Riesz space. These two Lebesgue decompositions usually differ from each other; for order countably additive vector measures they coincide if and only if the dimension of the Riesz space is equal to one.

In Section 3 we recall some known results on bounded vector measures and vector measures of bounded variation in a Banach space. In particular, we state the YosidaHewitt decomposition theorem of Uhl [14] and the Lebesgue decomposition theorem of Rickart [9] and Uhl [14] for vector measures of bounded variation. These results are included for reference and in the form they will be needed in Section 4.

In Section 4 we study vector measures of bounded variation in a Banach lattice having property $(P)$. As remarked before, the key result of this section is the Jordan decomposition theorem of [11], which generalizes results of Diestel and Faires [3] and Faires and Morrison [5]. The Jordan decomposition theorem is used to prove that the Yosida-Hewitt and Lebesgue decompositions are band decompositions. Furthermore, it is shown that the decomposition theorems of the Yosida-Hewitt type and those of the Lebesgue type differ considerably in the coincidence of their lattice theoretical and 
topological versions: In a weakly sequentially complete Banach lattice, the YosidaHewitt band decomposition and the Yosida-Hewitt decomposition (of Uhl) always coincide, whereas the Lebesgue band decomposition and the Lebesgue decomposition (of Rickart and Uhl) coincide if and only if the dimension of the Banach lattice is equal to one. For (order) countably additive vector measures, however, the Lebesgue null-set decomposition always coincides with the Lebesgue decomposition.

We conclude with some remarks and complements in Section 5.

Let us now fix some notation which will be used throughout this paper.

Let $\mathscr{F}$ be an algebra of subsets of some set $\Omega$. If the sets $A, B \in \mathscr{F}$ are disjoint, then their union will be denoted by $A+B$. For a set $A \in \mathscr{F}$, define $\mathscr{F}(A):=\{B \in \mathscr{F} \mid B \subseteq A\}$. A finite collection $\left\{A_{1}, A_{2}, \ldots, A_{k}\right\}$ of mutually disjoint sets in $\mathscr{F}(A)$ is a partition of $A$ in $\mathscr{F}$ if it satisfies $A=A_{1}+A_{2}+\cdots+A_{k}$. Let $\mathscr{P}(A)$ denote the class of all partitions of $A$ in $\mathscr{F}$. The characteristic function of $A$ will be denoted by $\chi_{A}$. A map $g: \Omega \rightarrow \mathbb{R}$ is a simple function if it can be written in the form $g=\alpha_{1} \chi_{A_{1}}+\alpha_{2} \chi_{A_{2}}+\cdots+\alpha_{k} \chi_{A_{k}}$, where $\left\{A_{1}, A_{2}, \ldots, A_{k}\right\}$ is a partition of $\Omega$ in $\mathscr{F}$ and $\alpha_{1}, \alpha_{2}, \ldots, \alpha_{k}$ are real scalars. Let $\mathbb{D}$ denote the sup-norm completion of the Riesz space of all simple functions $\Omega \rightarrow \mathbb{R}$, endowed with the canonical order. Then $\mathbb{D}$ is an $A M$-space with unit $\chi_{\Omega}$.

Let $\mathbb{E}$ be a real vector space. A set function $\mu: \mathscr{F} \rightarrow \mathbb{E}$ is additive or a vector measure if $\mu(A+B)=\mu(A)+\mu(B)$ holds for each pair of disjoint sets $A, B \in \mathscr{F}$. Let $a(\mathscr{F}, \mathbb{E})$ denote the class of all vector measures $\mathscr{F} \rightarrow \mathbb{E}$. For a vector measure $\mu \in a(\mathscr{F}, \mathbb{E})$ and a set $A \in \mathscr{F}$, let $R_{A} \mu$ denote the restriction of $\mu$ to $\mathscr{F}(A)$.

For detailed information on vector measures, Riesz spaces, and Banach lattices, we refer to the books by Diestel and Uhl [4], Luxemburg and Zaanen [6], and Schaefer [10].

\section{Vector measures in a Riesz space}

Throughout this section, suppose that $\mathbb{E}$ is an order complete Riesz space.

A vector measure $\mu \in a(\mathscr{F}, \mathbb{E})$ is positive if $\mu(A) \in \mathbb{E}_{+}$holds for all $A \in \mathscr{F}$, and it is order bounded if $\sup _{\mathscr{F}}|\mu(A)|$ exists in $\mathbb{E}$. Let $\operatorname{oba}(\mathscr{F}, \mathbb{E})$ denote the class of all order bounded vector measures in $a(\mathscr{F}, \mathbb{E})$. Endowed with the canonical order, the class $o b a(\mathscr{F}, \mathbb{E})$ is an ordered vector space. More precisely, we have the following result:

Theorem 2.1. The class oba $(\mathscr{F}, \mathbb{E})$ is an order complete Riesz space, and the identities

and

$$
(\mu \vee \phi)(A)=\sup _{\mathscr{F}(A)}(\mu(B)+\phi(A \backslash B))
$$

$$
(\mu \wedge \phi)(A)=\inf _{\mathscr{F}(A)}(\mu(B)+\phi(A \backslash B))
$$

hold for all $\mu, \phi \in o b a(\mathscr{F}, \mathbb{E})$ and $A \in \mathscr{F}$. Moreover, if $\left\{\mu_{\gamma} \in o b a(\mathscr{F}, \mathbb{E}) \mid \gamma \in \Gamma\right\}$ is a directed family which is bounded above, then

$$
\left(\sup _{\Gamma} \mu_{\gamma}\right)(A)=\sup _{\Gamma} \mu_{\gamma}(A)
$$

holds for all $A \in \mathscr{F}$. 
The proof of this result proceeds by standard arguments and is therefore omitted.

A vector measure has a Jordan decomposition if it is the difference of two orthogonal positive vector measures. As an immediate consequence of Theorem 2.1, we obtain the following Jordan decomposition theorem which is essentially due to Faires and Morrison [5]:

Corollary 2.2. A vector measure $\mu \in a(\mathscr{F}, \mathbb{E})$ is order bounded if and only if it has a Jordan decomposition. Moreover, if $\mu$ is order bounded, then its Jordan decomposition is unique and given by $\mu=\mu^{+}-\mu^{-}$.

A vector measure $\mu \in \operatorname{oba}(\mathscr{F}, \mathbb{E})$ is order countably additive if $\mu\left(\sum A_{n}\right)=o-\sum \mu\left(A_{n}\right)$ holds for each sequence $\left\{A_{n} \in \mathscr{F} \mid n \in \mathbb{N}\right\}$ of mutually disjoint sets satisfying $\sum A_{n} \in \mathscr{F}$. It is not hard to see that a vector measure $\mu \in o b a(\mathscr{F}, \mathbb{E})$ is order countably additive if and only if $o-\lim \mu\left(B_{n}\right)=0$ holds for each decreasing sequence $\left\{B_{n} \in \mathscr{F} \mid n \in \mathbb{N}\right\}$ satisfying $\bigcap_{N} B_{n}=\varnothing$. Let $o b c a(\mathscr{F}, \mathbb{E})$ denote the class of all order countably additive vector measures in $o b a(\mathscr{F}, \mathbb{E})$. A vector measure $\mu \in o b a(\mathscr{F}, \mathbb{E})$ is order purely finitely additive if $\phi=0$ holds for each vector measure $\phi \in o b c a(\mathscr{F}, \mathbb{E})$ satisfying $|\phi| \leqq|\mu|$. Let $\operatorname{obpfa}(\mathscr{F}, \mathbb{E})$ denote the class of all order purely finitely additive vector measures in $o b a(\mathscr{F}, \mathbb{E})$. We have the following Yosida-Hewitt band decomposition theorem which is essentially due to Congost Iglesias [2]:

Theorem 2.3. The classes obca $(\mathscr{F}, \mathbb{E})$ and obpfa $(\mathscr{F}, \mathbb{E})$ are order complete Riesz spaces and projection bands in oba $(\mathscr{F}, \mathbb{E})$, and oba $(\mathscr{F}, \mathbb{E})$ is the direct sum of obca $(\mathscr{F}, \mathbb{E})$ and obpfa $(\mathscr{F}, \mathbb{E})$. In particular, if $\mu \in o b a(\mathscr{F}, \mathbb{E})$ has the decomposition $\mu=\mu_{1}+\mu_{2}$, with $\mu_{1} \in \operatorname{obca}(\mathscr{F}, \mathbb{E})$ and $\mu_{2} \in \operatorname{obpfa}(\mathscr{F}, \mathbb{E})$, then $|\mu|=\left|\mu_{1}\right|+\left|\mu_{2}\right|,\left|\mu_{1}\right|=\sup [0,|\mu|] \cap o b c a(\mathscr{F}, \mathbb{E})$ and $\left|\mu_{2}\right|=\sup [0,|\mu|] \cap \operatorname{obpfa}(\mathscr{F}, \mathbb{E})$.

Proof. Let us first study the properties of the class $o b c a(\mathscr{F}, \mathbb{E})$. Consider $\mu \in o b a(\mathscr{F}, \mathbb{E})$. If $\mu$ is order countably additive and $\left\{A_{n} \in \mathscr{F} \mid n \in \mathbb{N}\right\}$ is a sequence of mutually disjoint sets satisfying $A:=\sum A_{n} \in \mathscr{F}$, then we have

$$
o-\sum|\mu|\left(A_{n}\right) \leqq|\mu|(A)
$$

and, for all $B \in \mathscr{F}$ and $B_{n}:=A_{n} \cap B$,

$$
\begin{aligned}
\mu(B)-\mu(A \backslash B) & =o-\sum \mu\left(B_{n}\right)-o-\sum \mu\left(A_{n} \backslash B_{n}\right) \\
& \leqq o-\sum|\mu|\left(B_{n}\right)+o-\sum|\mu|\left(A_{n} \backslash B_{n}\right) \\
& =o-\sum|\mu|\left(A_{n}\right),
\end{aligned}
$$

hence

$$
|\mu|(A) \leqq o-\sum|\mu|\left(A_{n}\right)
$$

which yields $|\mu| \in \operatorname{obca}(\mathscr{F}, \mathbb{E})$. Conversely, if $|\mu|$ is order countably additive, then the same 
is true for $\mu^{+}$and $\mu^{-}$, and hence for $\mu$. Therefore, the class $o b c a(\mathscr{F}, \mathbb{E})$ is a Riesz space and an ideal in $o b a(\mathscr{F}, \mathbb{E})$.

Furthermore, if $\left\{\mu_{\gamma} \in o b c a(\mathscr{F}, \mathbb{E}) \mid \gamma \in \Gamma\right\}$ is a directed family which is bounded above in $o b a(\mathscr{F}, \mathbb{E})$, then $\mu:=\sup _{\Gamma} \mu_{\gamma}$ exists in $o b a(\mathscr{F}, \mathbb{E})$, and we have $\mu(A)=\sup _{\mathrm{r}} \mu_{\gamma}(A)$, for all $A \in \mathscr{F}$. We may and do assume that

$$
0 \leqq \mu_{\gamma}
$$

holds for all $\gamma \in \Gamma$. Then we have, for all $\gamma \in \Gamma$ and for each sequence $\left\{A_{n} \in \mathscr{F} \mid n \in \mathbb{N}\right\}$ of mutually disjoint sets satisfying $A:=\sum A_{n} \in \mathscr{F}$,

$$
\mu_{\gamma}(A)=o-\sum \mu_{\gamma}\left(A_{n}\right) \leqq o-\sum \mu\left(A_{n}\right)
$$

hence

$$
\mu(A)=\sup _{\Gamma} \mu_{\gamma}(A) \leqq o-\sum \mu\left(A_{n}\right) \leqq \mu(A),
$$

which yields $\mu \in \operatorname{obca}(\mathscr{F}, \mathbb{E})$. Therefore, the class $\operatorname{obca}(\mathscr{F}, \mathbb{E})$ is even a band in $\operatorname{oba}(\mathscr{F}, \mathbb{E})$ and, in particular, an order complete Riesz space.

Let us now study the properties of the class $\operatorname{obpf} a(\mathscr{F}, \mathbb{E})$. Consider $\mu \in o b a(\mathscr{F}, \mathbb{E})$. If $\mu$ is order purely finitely additive, then we have, for all $\phi \in o b c a(\mathscr{F}, \mathbb{E}),|\mu| \wedge|\phi| \leqq|\phi|$, hence $|\mu| \wedge|\phi| \in \operatorname{obca}(\mathscr{F}, \mathbb{E})$, and $|\mu| \wedge|\phi| \leqq|\mu|$, which yields $|\mu| \wedge|\phi|=0$. Conversely, if $\mu$ is such that $|\mu| \wedge|\phi|=0$ holds for all $\phi \in o b c a(\mathscr{F}, \mathbb{E})$, then we have $\phi=0$ for all $\phi \in o b c a(\mathscr{F}, \mathbb{E})$

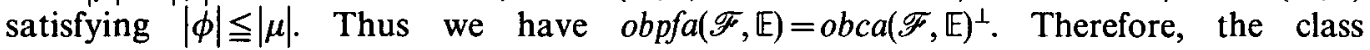
$o b p f a(\mathscr{F}, \mathbb{E})$ is a band in $o b a(\mathscr{F}, \mathbb{E})$ and, in particular, an order complete Riesz space.

Finally, since the Riesz space $o b a(\mathscr{F}, \mathbb{E})$ is order complete, the bands obca $(\mathscr{F}, \mathbb{E})$ and $\operatorname{obpfa}(\mathscr{F}, \mathbb{E})$ are projection bands, and $o b a(\mathscr{F}, \mathbb{E})$ is the direct sum of $o b c a(\mathscr{F}, \mathbb{E})$ and $\operatorname{obpfa}(\mathscr{F}, \mathbb{E})$.

For the remainder of this section, let $\phi \in o b a(\mathscr{F}, \mathbb{E})$ be a fixed vector measure and let $B(\{\phi\})$ denote the principal band generated by $\{\phi\}$.

A vector measure $\mu \in o b a(\mathscr{F}, \mathbb{E})$ is order $\phi$-continuous if $\mu \in B(\{\phi\})$, and it is order $\phi$-singular if $\mu \in\{\phi\}^{\perp}$. We have the following Lebesgue band decomposition theorem.

Theorem 2.4. The classes $B(\{\phi\})$ and $\{\phi\}^{\perp}$ are order complete Riesz spaces and

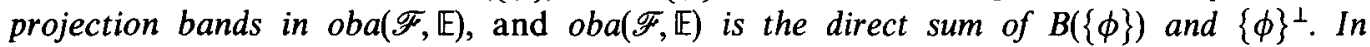
particular, if $\mu \in \operatorname{oba}(\mathscr{F}, \mathbb{E})$ has the decomposition $\mu=\mu_{1}+\mu_{2}$, with $\mu_{1} \in B(\{\phi\})$ and $\mu_{2} \in\{\phi\}^{\perp}$, then $|\mu|=\left|\mu_{1}\right|+\left|\mu_{2}\right|,\left|\mu_{1}\right|=\sup [0,|\mu|] \cap B(\{\phi\})$ and $\left|\mu_{2}\right|=\sup [0,|\mu|] \cap\{\phi\}^{\perp}$.

Proof. Since the Riesz space $o b a(\mathscr{F}, \mathbb{E})$ is order complete, the bands $B(\{\phi\})$ and $\{\phi\}^{\perp}$ are projection bands, and $o b a(\mathscr{F}, \mathbb{E})$ is the direct sum of $B(\{\phi\})$ and $\{\phi\}^{\perp}$.

Corollary 2.5. If $B$ is $a$ band in oba(F, $\mathbb{E})$ containing $\phi$, then every vector measure in oba $(\mathscr{F}, \mathbb{E})$ has a unique decomposition into an order $\phi$-continuous vector measure in $B$ and an order $\phi$-singular vector measure. 
In the case where $\phi$ is order countably additive, a different decomposition theorem of the Lebesgue type can be given under additional assumptions on $\mathscr{F}$ and $\mathbb{E}$. Let $c^{\phi}(\mathscr{F}, \mathbb{E})$ denote the class of all vector measures $\mu \in o b a(\mathscr{F}, \mathbb{E})$ for which $|\phi|(A)=0$ implies $|\mu|(A)=0$, and let $s^{\phi}(\mathscr{F}, \mathbb{E})$ denote the class of all vector measures $\mu \in o b a(\mathscr{F}, \mathbb{E})$ for which there exists a set $N \in \mathscr{F}$ satisfying $|\phi|(N)=0$ and $|\mu|(\Omega \backslash N)=0$. We have the following Lebesgue null-set decomposition theorem:

Theorem 2.6. Suppose $\mathbb{E}$ is super Dedekind complete, $\mathscr{F}$ is a $\sigma$-algebra, and $\phi$ is order countably additive. Then the classes $c^{\phi}(\mathscr{F}, \mathbb{E})$ and $s^{\phi}(\mathscr{F}, \mathbb{E})$ are order complete Riesz spaces and projection bands in oba $(\mathscr{F}, \mathbb{E})$ and oba $(\mathscr{F}, \mathbb{E})$ is the direct sum of $c^{\phi}(\mathscr{F}, \mathbb{E})$ and $s^{\phi}(\mathscr{F}, \mathbb{E})$. In particular, if $\mu \in \operatorname{oba}(\mathscr{F}, \mathbb{E})$ has the decomposition $\mu=\mu_{1}+\mu_{2}$, with $\mu_{1} \in c^{\phi}(\mathscr{F}, \mathbb{E})$ and $\mu_{2} \in s^{\phi}(\mathscr{F}, \mathbb{E}), \quad$ then $\quad|\mu|=\left|\mu_{1}\right|+\left|\mu_{2}\right|, \quad\left|\mu_{1}\right|=\sup [0,|\mu|] \cap c^{\phi}(\mathscr{F}, \mathbb{E}) \quad$ and $\quad\left|\mu_{2}\right|$ $=\sup [0,|\mu|] \cap s^{\phi}(\mathscr{F}, \mathbb{E})$.

Proof. The classes $c^{\phi}(\mathscr{F}, \mathbb{E})$ and $s^{\phi}(\mathscr{F}, \mathbb{E})$ are ideals in $o b a(\mathscr{F}, \mathbb{E})$, and we have $c^{\phi}(\mathscr{F}, \mathbb{E}) \cap s^{\phi}(\mathscr{F}, \mathbb{E})=\{0\}$. Let us now show that oba $\left.\mathscr{F}, \mathbb{E}\right)$ is the sum of these ideals. Consider $\mu \in o b a(\mathscr{F}, \mathbb{E})$. We may and do assume that $\phi$ and $\mu$ are positive.

Define $\mathscr{N}:=\{A \in \mathscr{F} \mid \phi(A)=0\}$. Since $\mathscr{F}$ is a $\sigma$-algebra and $\phi$ is order countably additive, the class $\mathscr{N}$ is a $\sigma$-algebra too. Clearly,

$$
z:=\sup _{\mathcal{N}} \mu(A)
$$

exists. Since $\mathbb{E}$ is super Dedekind complete, there exists a sequence $\left\{A_{n} \in \mathcal{N} \mid n \in \mathbb{N}\right\}$ satisfying

$$
z=\sup _{\mathrm{N}} \mu\left(A_{n}\right) .
$$

Define $N:=\bigcup_{N} A_{n}$. Then we have $N \in \mathcal{N}$ and, for all $n \in \mathbb{N}$,

$$
\mu\left(A_{n}\right) \leqq \mu(N) \leqq z,
$$

hence

$$
z=\mu(N)
$$

Now define, for all $A \in \mathscr{F}$,

$$
\mu_{1}(A):=\mu(A \cap(\Omega \backslash N))
$$

and

$$
\mu_{2}(A):=\mu(A \cap N)
$$

Then $\mu_{1}$ and $\mu_{2}$ are positive vector measures in oba(F्F, E) satisfying $\mu=\mu_{1}+\mu_{2}$. Furthermore, for all $A \in \mathcal{N}$, we have $N+A \cap(\Omega \backslash N) \in \mathcal{N}$, hence

$$
\mu(N) \leqq \mu(N)+\mu(A \cap(\Omega \backslash N))=\mu(N+A \cap(\Omega \backslash N)) \leqq z=\mu(N),
$$


which yields

$$
\mu_{1}(A)=\mu(A \cap(\Omega \backslash N))=0,
$$

and we also have

$$
\phi(N)+\mu_{2}(\Omega \backslash N)=0 .
$$

Therefore, we have $\mu_{1} \in c^{\phi}(\mathscr{F}, \mathbb{E})$ and $\mu_{2} \in s^{\phi}(\mathscr{F}, \mathbb{E})$.

Thus we have shown that $c^{\phi}(\mathscr{F}, \mathbb{E})$ and $s^{\phi}(\mathscr{F}, \mathbb{E})$ are complementary ideals in oba $(\mathscr{F}, \mathbb{E})$. It then follows that $c^{\phi}(\mathscr{F}, \mathbb{E})$ and $s^{\phi}(\mathscr{F}, \mathbb{E})$ are projection bands, and that $o b a(\mathscr{F}, \mathbb{E})$ is the direct sum of $c^{\phi}(\mathscr{F}, \mathbb{E})$ and $s^{\phi}(\mathscr{F}, \mathbb{E})$.

For the class of all order countably additive vector measures, the Lebesgue null-set decomposition theorem is essentially due to Pavlakos [8]; see also Congost Iglesias [2]. Their result is a consequence of Theorem 2.6 since $o b c a(\mathscr{F}, \mathbb{E})$ is a band in $o b a(\mathscr{F}, \mathbb{E})$.

More generally, all decomposition theorems of this section can be refined in the following way: If $B$ is a band in $\operatorname{oba}(\mathscr{F}, \mathbb{E})$ and $\mu$ is a vector measure in $B$, then $\mu^{+}$and $\mu^{-}$are contained in $B$, and if $\mu=\mu_{1}+\mu_{2}$ is the Yosida-Hewitt band decomposition, a Lebesgue band decomposition, or a Lebesgue null-set decomposition, of $\mu$, then $\mu_{1}$ and $\mu_{2}$ are contained in $B$.

Let us finally compare the Lebesgue band decomposition and the Lebesgue null-set decomposition. From the following result, it can be seen that these two Lebesgue decompositions usually differ from each other, except for order countably additive vector measures in the case where the dimension of $\mathbb{E}$ is equal to one:

Corollary 2.7. Suppose $\mathbb{E}$ is super Dedekind complete, $\mathscr{F}$ is a $\sigma$-algebra, and $\phi$ is order countably additive. Then $B(\{\phi\}) \subseteq c^{\phi}(\mathscr{F}, \mathbb{E})$ and $s^{\phi}(\mathscr{F}, \mathbb{E}) \subseteq\{\phi\}^{\perp}$. Moreover, the identities $B(\{\phi\})=c^{\phi}(\mathscr{F}, \mathbb{E}) \cap o b c a(\mathscr{F}, \mathbb{E})$ and $\{\phi\}^{\perp} \cap \operatorname{obca}(\mathscr{F}, \mathbb{E})=s^{\phi}(\mathscr{F}, \mathbb{E}) \cap$ obca $(\mathscr{F}, \mathbb{E})$ hold for each $\sigma$-algebra $\mathscr{F}$ and for each vector measure $\phi \in o b c a(\mathscr{F}, \mathbb{E})$ if and only if the dimension of $\mathbb{E}$ is equal to one.

Proof. We clearly have $s^{\phi}(\mathscr{F}, \mathbb{E}) \subseteq\{\phi\}^{\perp}$. By the Lebesgue null-set decomposition, each vector measure $\mu \in B(\{\phi\})$ is the sum of a vector measure $\mu_{1} \in c^{\phi}(\mathscr{F}, \mathbb{E}) \cap B(\{\phi\})$ and a vector measure $\mu_{2} \in s^{\phi}(\mathscr{F}, \mathbb{E}) \cap B(\{\phi\}) \subseteq\{\phi\}^{\perp} \cap B(\{\phi\})=\{0\}$. Thus we have $B(\{\phi\}) \subseteq c^{\phi}(\mathscr{F}, \mathbb{E})$.

Now suppose that the dimension of $\mathbb{E}$ is equal to one. Consider $\mu \in\{\phi\}^{\perp} \cap \operatorname{obca}(\mathscr{F}, \mathbb{E})$ and define $\psi:=|\phi|-|\mu|$. Then we have $\psi^{+}=|\phi|$ and $\psi^{-}=|\mu|$. By the Hahn decomposition of $\psi$, there exists a set $N \in \mathscr{F}$ satisfying $|\phi|(N)+|\mu|(\Omega \backslash N)=0$, which yields $\mu \in s^{\phi}(\mathscr{F}, \mathbb{E})$. Thus we have $\{\phi\}^{\perp} \cap \operatorname{obca}(\mathscr{F}, \mathbb{E})=s^{\phi}(\mathscr{F}, \mathbb{E}) \cap o b c a(\mathscr{F}, \mathbb{E})$, and consequently $B(\{\phi\})=c^{\phi}(\mathscr{F}, \mathbb{E}) \cap \operatorname{obca}(\mathscr{F}, \mathbb{E})$.

Finally, suppose that the dimension of $\mathbb{E}$ is greater than one. Then $\mathbb{E}$ fails to be totally ordered, and it follows from the subsequent Example 2.8 that there exists a $\sigma$-algebra $\mathscr{F}$ and a vector measure $\phi \in o b c a(\mathscr{F}, \mathbb{E})$ satisfying $c^{\phi}(\mathscr{F}, \mathbb{E}) \cap\{\phi\}^{\perp} \cap o b c a(\mathscr{F}, \mathbb{E}) \neq\{0\}$.

Example 2.8. Consider a super Dedekind complete Riesz space $\mathbb{E}$ which fails to be totally ordered. Then there exist $x_{1}, x_{2} \in \mathbb{E}$ satisfying $x_{1} \not \leq x_{2}$ and $x_{2} \not \leq x_{1}$. Define $e_{1}:=$ 
$\left(x_{1}-x_{2}\right)$ and $e_{2}:=\left(x_{2}-x_{1}\right)^{+}$. Then we have $e_{1} \wedge e_{2}=0$. Let $\mathscr{F}$ denote the $\sigma$-algebra consisting of all Borel subsets of the interval $[0,1]$, and let $\lambda: \mathscr{F} \rightarrow[0,1]$ denote the Lebesgue measure on $\mathscr{F}$. For all $A \in \mathscr{F}$, define

$$
\phi(A):=\lambda(A) e_{1}
$$

and

$$
\mu(A):=\lambda(A) e_{2}
$$

Then $\phi$ and $\mu$ are positive vector measures in $\operatorname{obca}(\mathscr{F}, \mathbb{E})$, and we have $\mu \in c^{\phi}(\mathscr{F}, \mathbb{E}) \cap\{\phi\}^{\perp} \cap o b c a(\mathscr{F}, \mathbb{E})$. In addition, we remark that the vector measure $\phi-\mu$ does not possess a Hahn decomposition.

\section{Vector measures in a Banach space}

Throughout this section, suppose that $\mathbb{E}$ is a Banach space.

The semivariation $\|\mu\| \|: \mathscr{F} \rightarrow \mathbb{B}_{+}$of a vector measure $\mu \in a(\mathscr{F}, \mathbb{E})$ is defined by letting

$$
\|\mu\| \mid(A):=\sup \left\|\sum \alpha_{i} \mu\left(A_{i}\right)\right\|
$$

for all $A \in \mathscr{F}$, where the supremum is taken over all partitions $\left\{A_{1}, A_{2}, \ldots, A_{k}\right\} \in \mathscr{P}(A)$ and scalars $\alpha_{1}, \alpha_{2}, \ldots, \alpha_{k} \in[-1,1]$. For all $A \in \mathscr{F}$, we have

$$
\sup _{\mathscr{F}(A)}\|\mu(B)\| \leqq\|\mu\|\left\|(A) \leqq 2 \sup _{\mathscr{F}(A)}\right\| \mu(B) \|
$$

A vector measure $\mu \in a(\mathscr{F}, \mathbb{E})$ is bounded if the value $\|\mu\|(\Omega)$ is finite. Let $b a(\mathscr{F}, \mathbb{E})$ denote the class of all bounded vector measures in $a(\mathscr{F}, \mathbb{E})$.

Let $\mathscr{L}(\mathbb{D}, \mathbb{E})$ denote the Banach space of all bounded linear operators $\mathbb{D} \rightarrow \mathbb{E}$, endowed with the operator norm. For each vector measure $\mu \in b a(\mathscr{F}, \mathbb{E})$, there exists a unique linear operator $T \in \mathscr{L}(\mathbb{D}, \mathbb{E})$ satisfying

$$
T\left(\sum \alpha_{i} \chi_{A_{i}}\right)=\sum \alpha_{i} \mu\left(A_{i}\right)
$$

for each simple function $\sum \alpha_{i} \chi_{A_{i}} \in \mathbb{D}$. This linear operator is called the representing linear operator of $\mu$. Let

$$
\mathscr{X}: b a(\mathscr{F}, \mathbb{E}) \rightarrow \mathscr{L}(\mathbb{D}, \mathbb{E})
$$

denote the map associating with each vector measure in $b a(\mathscr{F}, \mathbb{E})$ its representing linear operator. We have the following representation theorem for bounded vector measures:

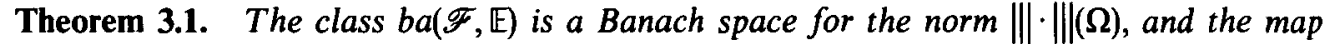
$\mathscr{X}$ is an isometric isomorphism of ba(F, $\mathbb{E})$ onto $\mathscr{L}(\mathbb{D}, \mathbb{E})$.

The variation $\|\mu\|: \mathscr{F} \rightarrow \mathbb{R}_{+}$of a vector measure $\mu \in a(\mathscr{F}, \mathbb{E})$ is defined by letting

$$
\|\mu\|(A):=\sup _{\mathscr{P}(A)} \sum\left\|\mu\left(A_{i}\right)\right\|
$$


for all $A \in \mathscr{F}$. A vector measure $\mu \in a(\mathscr{F}, \mathbb{E})$ has bounded variation if the value $\|\mu\|(\Omega)$ is finite. Let $b v a(\mathscr{F}, \mathbb{E})$ denote the class of all vector measures of bounded variation in $a(\mathscr{F}, \mathbb{E})$.

Let $\mathscr{L}^{l}(\mathbb{D}, \mathbb{E})$ denote the Banach space of all cone absolutely summing operators $\mathbb{D} \rightarrow \mathbb{E}$, endowed with the norm $\|\cdot\|_{t}$ which is given by

$$
\|T\|_{l}:=\sup \sum\left\|T x_{i}\right\|,
$$

where the supremum is taken over all finite collections $\left\{x_{1}, x_{2}, \ldots, x_{k}\right\} \subseteq \mathbb{D}+$ satisfying $\left\|\sum x_{i}\right\|=1$. We have the following representation theorem for vector measures of bounded variation in a Banach space:

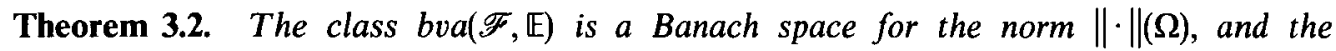
restriction of $\mathscr{X}$ is an isometric isomorphism of bva $(\mathscr{F}, \mathbb{E})$ onto $\mathscr{L}^{l}(\mathbb{D}, \mathbb{E})$. In particular, \|\|$\mu\|(\Omega) \leqq\| \mu \|(\Omega)$ holds for all $\mu \in \operatorname{bva}(\mathscr{F}, \mathbb{E})$.

In the previous result, the Banach space $\mathscr{L}^{\prime}(\mathbb{D}, \mathbb{E})$ can be replaced by the Banach space of all absolutely summing operators $\mathbb{D} \rightarrow \mathbb{E}$. This is due to the fact that $\mathbb{D}$ is an $A M$-space.

A vector measure $\mu \in a(\mathscr{F}, \mathbb{E})$ is countably additive if $\mu\left(\sum A_{n}\right)=\sum \mu\left(A_{n}\right)$ holds for each sequence $\left\{A_{n} \in \mathscr{F} \mid n \in \mathbb{N}\right\}$ of mutually disjoint sets satisfying $\sum A_{n} \in \mathscr{F}$. A vector measure $\mu \in b v a(\mathscr{F}, \mathbb{E})$ is countably additive if and only if its variation $\|\mu\|$ is countably additive. Let $b v c a(\mathscr{F}, \mathbb{E})$ denote the class of all countably additive vector measures in $b v a(\mathscr{F}, \mathbb{E})$. A vector measure $\mu \in b v a(\mathscr{F}, \mathbb{E})$ is purely finitely additive if $\phi=0$ holds for each vector measure $\phi \in b v c a(\mathscr{F}, \mathbb{E})$ satisfying $\|\phi\| \leqq\|\mu\|$. Let $b v p f a(\mathscr{F}, \mathbb{E})$ denote the class of all purely finitely additive vector measures in $b v a(\mathscr{F}, \mathbb{E})$. We have the following Yosida-Hewitt decomposition theorem which is due to $\mathrm{Uhl}$ [14]:

Theorem 3.3. The classes bvca $(\mathscr{F}, \mathbb{E})$ and bvpfa $(\mathscr{F}, \mathbb{E})$ are Banach spaces, and bva $(\mathscr{F}, \mathbb{E})$ is the direct sum of bvca $(\mathscr{F}, \mathbb{E})$ and bvfa $(\mathscr{F}, \mathbb{E})$. In particular, if $\mu \in b v a(\mathscr{F}, \mathbb{E})$ has the decomposition $\mu=\mu_{1}+\mu_{2}$, with $\mu_{1} \in \operatorname{bvca}(\mathscr{F}, \mathbb{E})$ and $\mu_{2} \in b v p f a(\mathscr{F}, \mathbb{E})$, then $\|\mu\|=\left\|\mu_{1}\right\|+\left\|\mu_{2}\right\|$.

For the remainder of this section, let $\lambda \in b a(\mathscr{F}, \mathbb{R})$ be a fixed (real-valued) bounded additive set function. A vector measure $\mu \in b v a(\mathscr{F}, \mathbb{E})$ is $\lambda$-continuous if $\|\mu\| \in B(\{\lambda\})$, and it is $\lambda$-singular if $\|\mu\| \in\{\lambda\}^{\perp}$. These definitions are in accordance with those given in [14] and [4]; see [12; Lemma 3.1.8]. Let $b v a^{\lambda \mathcal{c}}(\mathscr{F}, \mathbb{E})$ denote the class of all $\lambda$-continuous vector measures in $b v a(\mathscr{F}, \mathbb{E})$, and let $b v a^{\lambda s}(\mathscr{F}, \mathbb{E})$ denote the class of all $\lambda$-singular vector measures in bva $(\mathscr{F}, \mathbb{E})$. We have the following Lebesgue decomposition theorem which is due to Rickart [9] and Uhl [14]:

Theorem 3.4. The classes $b v a^{\lambda c}(\mathscr{F}, \mathbb{E})$ and $b v a^{\lambda s}(\mathscr{F}, \mathbb{E})$ are Banach spaces, and bva $(\mathscr{F}, \mathbb{E})$ is the direct sum of $b v a^{\lambda c}(\mathscr{F}, \mathbb{E})$ and $b v a^{\lambda s}(\mathscr{F}, \mathbb{E})$. In particular, if $\mu \in b v a(\mathscr{F}, \mathbb{E})$ has the decomposition $\mu=\mu_{1}+\mu_{2}$, with $\mu_{1} \in b v a^{\lambda c}(\mathscr{F}, \mathbb{E})$ and $\mu_{2} \in b v a^{\lambda s}(\mathscr{F}, \mathbb{E})$, then $\|\mu\|=\left\|\mu_{1}\right\|+\left\|\mu_{2}\right\|$.

The previous decomposition theorems can be proven by a Stone space argument. For the details, see [14] and [4]. 


\section{Vector measures in a Banach lattice}

Throughout this section, suppose that $\mathbb{E}$ is an order complete Banach lattice.

Let $\mathscr{L}^{r}(\mathbb{D}, \mathbb{E})$ denote the order complete Banach lattice of all regular operators $\mathbb{D} \rightarrow \mathbb{E}$, endowed with the canonical order and the norm $\|\cdot\|_{r}$ which is given by

$$
\|T\|_{r}:=\||T|\|
$$

see [10; Proposition IV.1.4]. We have the following representation theorem for order bounded vector measures:

Theorem 4.1. The class oba $(\mathscr{F}, \mathbb{E})$ is an order complete Banach lattice for the norm $\||\cdot|(\Omega)\|$, and the restriction of $\mathscr{X}$ is an isometric vector lattice isomorphism of oba $(\mathscr{F}, \mathbb{E})$ onto $\mathscr{L}^{r}(\mathbb{D}, \mathbb{E})$. In particular, $\||\mu\|(\Omega) \leqq\|\||\mu|\|(\Omega)=\||\mu|(\Omega)\|$ holds for all $\mu \in$ oba $(\mathscr{F}, \mathbb{E})$.

Proof. It is clear that each order bounded vector measure is bounded, and it is not hard to verify that a bounded vector measure is order bounded if and only if its representing linear operator is regular. For the details, see [12; Theorem 4.1.2].

For a large class of Banach lattices, we shall now study the decomposition properties of vector measures of bounded variation. The following representation theorem for vector measures of bounded variation in a Banach lattice having property $(P)$ will be essential in all further investigations of this section:

Theorem 4.2. Suppose $\mathbb{E}$ has property $(P)$. Then the class bva(F्F, $\mathbb{E})$ is an order complete Banach lattice for the norm $\|\cdot\|(\Omega)$ and an ideal in oba $(\mathscr{F}, \mathbb{E})$, and the restriction of $\mathscr{X}$ is an isometric vector lattice isomorphism of bva $(\mathscr{F}, \mathbb{E})$ onto $\mathscr{L}^{l}(\mathbb{D}, \mathbb{E})$. In particular, $\||\mu|(\Omega)\| \leqq\||\mu|\|(\Omega)=\|\mu\|(\Omega)$ holds for all $\mu \in b v a(\mathscr{F}, \mathbb{E})$.

Proof. Since $\mathbb{E}$ has property $(P)$, the class $\mathscr{L}^{\prime}(\mathbb{D}, \mathbb{E})$, endowed with the canonical order and the norm $\|\cdot\|_{l}$, is an order complete Banach lattice and an ideal in $\mathbb{L}(\mathbb{D}, \mathbb{E})$; see [10; Theorem IV.4.3]. Now the assertion follows from Theorem 3.2 and Theorem 4.1.

As an immediate consequence of Theorem 4.2, we obtain the following general Jordan decomposition theorem [11]:

Corollary 4.3. Suppose $\mathbb{E}$ has property $(P)$. Then each vector measure $\mu \in$ bva $(\mathscr{F}, \mathbb{E})$ is the difference of two orthogonal positive vector measures in bva(F, $\mathbb{E})$. Moreover, the decomposition is unique and given by $\mu=\mu^{+}-\mu^{-}$.

Since $A L$-spaces and, more generally, $K B$-spaces have property $(P)$, the previous result generalizes the Jordan decomposition theorems obtained by Diestel and Faires [3] and by Faires and Morrison [5]. This generalization is a proper one since dual Banach lattices and order complete $A M$-spaces with unit have property $(P)$. The Jordan decomposition of vector measures in an $A M$-space will be discussed in Section 5. 
Corollary 4.4. The following are equivalent:

(a) $\mathbb{E}$ is an AL-space.

(b) bva $(\mathscr{F}, \mathbb{E})$ is an $A L$-space.

Moreover, if $\mathbb{E}$ is an $A L$-space, then the Banach lattices bva $(\mathscr{F}, \mathbb{E})$ and oba $(\mathscr{F}, \mathbb{E})$ are identical.

Proof. Since $\mathbb{D}$ is an $A M$-space, the assertion follows from Theorem 4.2, Theorem 4.1, and [10; Proposition IV.4.5].

In general, however, the Banach lattice $b v a(\mathscr{F}, \mathbb{E})$ need not inherit the properties of $\mathbb{E}$. For example, there exists a $K B$-space $\mathbb{E}$ such that, for the $\sigma$-algebra $\mathscr{F}$ consisting of all Borel subsets of the interval $[0,1]$, the Banach lattice bva(F, $\mathbb{E})$ fails to be order continuous and thus cannot be a $K B$-space; see Talagrand [13]. Furthermore, the class $b v a(\mathscr{F}, \mathbb{E})$ can be strictly smaller than $o b a(\mathscr{F}, \mathbb{E})$. More precisely, the ideal $b v a(\mathscr{F}, \mathbb{E})$ may fail to be a $\sigma$-ideal in $o b a(\mathscr{F}, \mathbb{E})$, as can be seen from the following Example 4.5:

Example 4.5. Consider the Banach lattice $l^{\infty}$, endowed with the canonical order and the sup-norm, and let $\left\{e_{n} \mid n \in \mathbb{N}\right\}$ denote its canonical basis. Note that $l^{\infty}$ is an order complete $A M$-space with unit and therefore has property $(P)$. Let $\mathscr{F}$ denote the $\sigma$-algebra consisting of all subsets of $\mathbb{N}$. For all $n \in \mathbb{N}$ and $A \in \mathscr{F}$, define

$$
\mu_{n}(A):=\sum_{A \cap\{1, \ldots, n\}} e_{j}
$$

Then each $\mu_{n}$ is a positive vector measure in bva $\left(\mathscr{F}, l^{\infty}\right)$, and the sequence $\left\{\mu_{n} \in b v a\left(\mathscr{F}, l^{\infty}\right) \mid n \in \mathbb{N}\right\}$ increases to a vector measure $\mu \in o b a\left(\mathscr{F}, l^{\infty}\right)$ satisfying $\|\mu\|(\Omega)=\infty$.

In particular, the ideal bva $\mathscr{F}, \mathbb{E})$ need not be a band in $o b a(\mathscr{F}, \mathbb{E})$, and the order

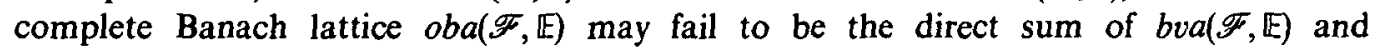
$\operatorname{bva}(\mathscr{F}, \mathbb{E})^{\perp}$.

Let us next study the Yosida-Hewitt decomposition.

Lemma 4.6. Suppose $\mathbb{E}$ has property $(P)$. Then, for each vector measure $\mu \in b v a(\mathscr{F}, \mathbb{E})$, the following are equivalent:

(a) $\mu$ is countably additive.

(b) $|\mu|$ is countably additive.

(c) $\|\mu\|$ is countably additive.

Proof. The equivalence of (a) and (c) is well-known. Consider $A \in \mathscr{F}$. Then $R_{A} \mu$ and 
$R_{A}|\mu|$ are vector measures in $b v a(\mathscr{F}(A), \mathbb{E})$, and we have $\left|R_{A} \mu\right|=R_{A}|\mu|$. By Theorem 4.2, $\operatorname{bva}(\mathscr{F}(A), \mathbb{E})$ is a Banach lattice for the norm $\|\cdot\|(A)$, which yields

$$
\||\mu|\|(A)=\left\|R_{A}|\mu|\right\|(A)=\left\|R_{A} \mu\right\|\|(A)=\| R_{A} \mu\|(A)=\| \mu \|(A) .
$$

Thus we have $\||\mu|\|=\|\mu\|$, and the equivalence of (b) and (c) now follows from the equivalence of (a) and (c), applied to the vector measure $|\mu| \in b v a(\mathscr{F}, \mathbb{E})$.

The following result can be proven by using similar arguments:

Lemma 4.7. Suppose $\mathbb{E}$ has property $(P)$. Then, for each vector measure $\mu \in$ bva $(\mathscr{F}, \mathbb{E})$, the following are equivalent:

(a) $\mu$ is purely finitely additive.

(b) $|\mu|$ is purely finitely additive.

(c) $\|\mu\|$ is purely finitely additive.

We can now improve the Yosida-Hewitt decomposition theorem:

Theorem 4.8. Suppose $\mathbb{E}$ has property $(P)$. Then the classes bvca $(\mathscr{F}, \mathbb{E})$ and bvpfa $(\mathscr{F}, \mathbb{E})$. are order complete Banach lattices and projection bands in bva $(\mathscr{F}, \mathbb{E})$, and bva $(\mathscr{F}, \mathbb{E})$ is the direct sum of bvca $(\mathscr{F}, \mathbb{E})$ and bvpfa(F, $\mathscr{E})$. In particular, the associated band projections are continuous, and if $\mu \in \operatorname{bva}(\mathscr{F}, \mathbb{E})$ has the decomposition $\mu=\mu_{1}+\mu_{2}$, with $\mu_{1} \in \operatorname{bvca}(\mathscr{F}, \mathbb{E})$ and $\mu_{2} \in \operatorname{bvpfa}(\mathscr{F}, \mathbb{E})$, then $|\mu|=\left|\mu_{1}\right|+\left|\mu_{2}\right|$ and $\|\mu\|=\left\|\mu_{1}\right\|+\left\|\mu_{2}\right\|$.

Proof. By Theorem 3.3 and Lemma 4.6, the class bvca $(\mathscr{F}, \mathbb{E})$ is a Banach lattice and an ideal in $b v a(\mathscr{F}, \mathbb{E})$.

If $\left\{\mu_{\gamma} \in b v c a(\mathscr{F}, \mathbb{E}) \mid \gamma \in \Gamma\right\}$ is a family of positive vector measures which are bounded above by $\phi \in b v c a(\mathscr{F}, \mathbb{E})$, then $\mu:=\sup _{\Gamma} \mu_{\gamma}$ exists in $b v a(\mathscr{F}, \mathbb{E})$. From $0 \leqq \mu \leqq \phi$ and the ideal property of $b v c a(\mathscr{F}, \mathbb{E})$, it follows that $\mu$ is countably additive. Thus $b v c a(\mathscr{F}, \mathbb{E})$ is an order complete Banach lattice, and the same is true for $b v p f a(\mathscr{F}, \mathbb{E})$. By Theorem 3.3,

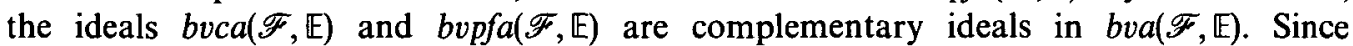
bva $(\mathscr{F}, \mathbb{E})$ is order complete, these complementary ideals are even projection bands, and $b v a(\mathscr{F}, \mathbb{E})$ is the direct sum of $b v c a(\mathscr{F}, \mathbb{E})$ and $b v p f a(\mathscr{F}, \mathbb{E})$. The continuity of the associated band projections is due to the fact that $b v a(\mathscr{F}, \mathbb{E})$ is a Banach lattice.

Corollary 4.9. Suppose $\mathbb{E}$ is a $K B$-space. If $\mu$ is a vector measure in bva( $\mathscr{F}, \mathbb{E})$, then

(a) $\mu$ is countably additive if and only if $\mu$ is order countably additive, and

(b) $\mu$ is purely finitely additive if and only if $\mu$ is order purely finitely additive.

Proof. Note that $\mathbb{E}$ is an order continuous Banach lattice having property $(P)$. By the Jordan decomposition, we may and do assume that $\mu$ is positive. 
If $\mu$ is countably additive, then it is order countably additive, by [10; Lemma II.5.8]. Conversely, if $\mu$ is order countably additive, then it is countably additive since $\mathbb{E}$ is order continuous.

If $\mu$ is purely finitely additive and $\phi$ is a vector measure in $o b c a(\mathscr{F}, \mathbb{E})$ satisfying $|\phi| \leqq \mu$, then we have $\|\phi\| \leqq\|\mu\|$, hence $\phi=0$, which means that $\mu$ is order purely finitely additive. Conversely, if $\mu$ is order purely finitely additive, then $\mu \wedge|\phi|=0$ holds for all $\phi \in b v c a(\mathscr{F}, \mathbb{E}) \subseteq o b c a(\mathscr{F}, \mathbb{E})$, by (a), which means that $\mu$ is purely finitely additive, by Theorem 4.8.

Thus, for a vector measure of bounded variation in a $K B$-space, the Yosida-Hewitt band decomposition and the Yosida-Hewitt decomposition are the same.

Let us now study the Lebesgue decomposition. For the remainder of this section, let $\lambda \in b a(\mathscr{F}, \mathbb{R})$ be a fixed (real-valued) bounded additive set function.

Lemma 4.10. Suppose $\mathbb{E}$ has property $(P)$. Then, for each vector measure $\mu \in$ bva $(\mathscr{F}, \mathbb{E})$, the following are equivalent:

(a) $\mu$ is $\lambda$-continuous.

(b) $|\mu|$ is $\lambda$-continuous.

(c) $\|\mu\|$ is $\lambda$-continuous.

Lemma 4.11. Suppose $\mathbb{E}$ has property $(P)$. Then, for each vector measure $\mu \in$ bva $(\mathscr{F}, \mathbb{E})$, the following are equivalent:

(a) $\mu$ is $\lambda$-singular.

(b) $|\mu|$ is $\lambda$-singular.

(c) $\|\mu\| u s$ isingular.

These two results can be proven in the same way as Lemma 4.6, and the arguments used in the proof of Theorem 4.8 also yield the following improved Lebesgue decomposition theorem:

Theorem 4.12. Suppose $\mathbb{E}$ has property $(P)$. Then the classes bva ${ }^{2 c}(\mathscr{F}, \mathbb{E})$ and bva ${ }^{\lambda s}(\mathscr{F}, \mathbb{E})$ are order complete Banach lattices and projection bands in bva $(\mathscr{F}, \mathbb{E})$, and bva $(\mathscr{F}, \mathbb{E})$ is the direct sum of $b v a^{\lambda c}(\mathscr{F}, \mathbb{E})$ and bva $a^{\lambda s}(\mathscr{F}, \mathbb{E})$. In particular, the associated band projections are continuous, and if $\mu \in b v a(\mathscr{F}, \mathbb{E})$ has the decomposition $\mu=\mu_{1}+\mu_{2}$, with $\mu_{1} \in b v a^{\lambda c}(\mathscr{F}, \mathbb{E})$ and $\mu_{2} \in b v a^{\lambda s}(\mathscr{F}, \mathbb{E})$, then $|\mu|=\left|\mu_{1}\right|+\left|\mu_{2}\right|$ and $\|\mu\|=\left\|\mu_{1}\right\|+\left\|\mu_{2}\right\|$.

For the remainder of this section, let $\phi \in b v a(\mathscr{F}, \mathbb{E})$ be a fixed vector measure. The following result, when compared with Corollary 4.9 , reveals a remarkable difference between the decomposition theorems of the Yosida-Hewitt type and those of the Lebesgue type: 
Corollary 4.13. Suppose $\mathbb{E}$ has property $(P)$. Then

(a) each $\|\phi\|$-singular vector measure in bva $(\mathscr{F}, \mathbb{E})$ is order $\phi$-singular, and

(b) each order $\phi$-continuous vector measure in bva $(\mathscr{F}, \mathbb{E})$ is $\|\phi\|$-continuous.

Moreover, the converse implications hold for each algebra $\mathscr{F}$ and for each vector measure $\phi \in b v a(\mathscr{F}, \mathbb{E})$ if and only if the dimension of $\mathbb{E}$ is equal to one.

Proof. Consider $\mu \in b v a(\mathscr{F}, \mathbb{E})$. For all $A \in \mathscr{F}$, we have

$$
\begin{aligned}
\||\mu| \wedge|\phi|||(\Omega) & =\||\mu| \wedge|\phi|\|(A)+\||\mu| \wedge|\phi|\|(\Omega \backslash A) \\
& \leqq\|\mu\|(A)+\|\phi\|(\Omega \backslash A)
\end{aligned}
$$

which yields

$$
\||\mu| \wedge|\phi|\|(\Omega) \leqq(\|\mu\| \wedge\|\phi\|)(\Omega)
$$

Therefore, $\|\mu\| \wedge\|\phi\|=0$ implies $|\mu| \wedge|\phi|=0$, which proves (a). If $\mu$ is order $\phi$ continuous, then $|\mu| \wedge|\psi|=0$ holds for all $\psi \in\{\phi\}^{\perp}$. In particular, by (a), we have $|\mu| \wedge|\psi|=0$ for all $\psi \in b v a^{\|\phi\| \mid s}(\mathscr{F}, \mathbb{E})$. Now it follows from Theorem 4.12 that $\mu$ is $\|\phi\|-$ continuous, which proves (b).

Now suppose that the dimension of $\mathbb{E}$ is equal to one. Then the modulus and the variation of a vector measure in $b v a(\mathscr{F}, \mathbb{E})$ coincide, which means that the implications in (a) and (b) can be reversed.

Finally, suppose that the dimension of $\mathbb{E}$ is greater than one. Then $\mathbb{E}$ fails to be totally ordered, and it follows from the subsequent Example 4.14 that there exists an algebra $\mathscr{F}$ and a vector measure $\phi \in b v a(\mathscr{F}, \mathbb{E})$ satisfying $b v a^{\|\phi\| c}(\mathscr{F}, \mathbb{E}) \cap\{\phi\}^{\perp} \neq\{0\}$.

Example 4.14. Consider a Banach lattice $\mathbb{E}$ having property $(P)$ which fails to be totally ordered. Then there exist $x_{1}, x_{2} \in \mathbb{E}$ satisfying $x_{1} \leq x_{2}$ and $x_{2} \leq x_{1}$. Define $e_{1}:=$ $\left(x_{1}-x_{2}\right)^{+}$and $e_{2}:=\left(x_{2}-x_{1}\right)^{+}$. Then we have $e_{1} \wedge e_{2}=0$. Let $\mathscr{F}$ denote the $\sigma$-algebra consisting of all Borel subsets of the interval $[0,1]$, and let $\lambda: \mathscr{F} \rightarrow[0,1]$ denote the Lebesgue measure on $\mathscr{F}$. For all $A \in \mathscr{F}$, define

$$
\phi(A):=\lambda(A) e_{1}
$$

and

$$
\mu(A):=\lambda(A) e_{2}
$$

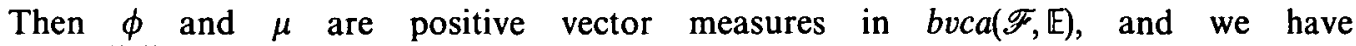
$\mu \in b v a^{\|\phi\| c}(\mathscr{F}, \mathbb{E}) \cap\{\phi\}^{\perp}$.

Thus, for a vector measure $\mu$ of bounded variation in a Banach lattice $\mathbb{E}$ having property $(P)$, the Lebesgue band decomposition of $\mu$ with respect to $\phi$ usually differs from the Lebesgue decomposition of $\mu$ with respect to $\|\phi\|$, except for the case where the dimension of $\mathbb{E}$ is equal to one. 
The following results will be used to compare the Lebesgue null-set decomposition and the Lebesgue decomposition:

Lemma 4.15. Suppose $\mathbb{E}$ has property $(P), \mathscr{F}$ is a $\sigma$-algebra, and $\phi$ is countably additive. Then, for each vector measure $\mu \in b v c a(\mathscr{F}, \mathbb{E})$, the following are equivalent:

(a) $\mu$ is $\|\phi\|$-continuous.

(b) $\|\phi\|(A)=0$ implies $\|\mu\|(A)=0$.

(c) $|\phi|(A)=0$ implies $|\mu|(A)=0$.

Proof. The equivalence of (a) and (b) is well-known, and the equivalence of (b) and (c) is obvious.

Lemma 4.16. Suppose $\mathbb{E}$ has property $(P), \mathscr{F}$ is a $\sigma$-algebra, and $\phi$ is countably additive. Then, for each vector measure $\mu \in b v c a(\mathscr{F}, \mathbb{E})$, the following are equivalent:

(a) $\mu$ is $\|\phi\|$-singular.

(b) There exists a set $N \in \mathscr{F}$ satisfying $\|\phi\|(N)+\|\mu\|(\Omega \backslash N)=0$.

(c) There exists a set $N \in \mathscr{F}$ satisfying $|\phi|(N)+|\mu|(\Omega \backslash N)=0$.

Proof. The equivalence of (a) and (b) is immediate from the Hahn decomposition of $\|\phi\|-\|\mu\|$, and the equivalence of (b) and (c) is obvious.

Thus, for a countably additive vector measure $\mu$ of bounded variation on a $\sigma$-algebra $\mathscr{F}$ and in a $K B$-space $\mathbb{E}$ (which is super Dedekind complete and has property $(P)$ ), the Lebesgue null-set decomposition of $\mu$ with respect to $\phi \in b v c a(\mathscr{F}, \mathbb{E})$ and the Lebesgue decomposition of $\mu$ with respect to $\|\phi\|$ are the same.

We finally remark that the Yosida-Hewitt decomposition theorem and the Lebesgue decomposition theorem can be refined for bands in $b v a(\mathscr{F}, \mathbb{E})$ in the same way as explained in Section 2.

\section{Remarks}

In the Jordan decomposition theorem for vector measures of bounded variation, it is not sufficient to assume that the Banach lattice $\mathbb{E}$ is order complete. This can be seen from the following Example 5.1:

Example 5.1. Consider the Banach lattice $c_{0}$, endowed with the canonical order and the sup-norm, and let $\left\{e_{n} \mid n \in \mathbb{N}\right\}$ denote its canonical basis. Note that $c_{0}$ is an order complete $A M$-space which does not possess a unit and therefore does not have property $(P)$. Let $\mathscr{F}$ denote the $\sigma$-algebra consisting of all Borel subsets of the interval $[0,2 \pi]$, and let $\lambda: \rightarrow[0,2 \pi]$ denote the Lebesgue measure on $\mathscr{F}$. For all $n \in \mathbb{N}$ and $A \in \mathscr{F}$, define

$$
\lambda_{n}(A):=\int_{A} \sin n t d \lambda(t)
$$


and, for all $A \in \mathscr{F}$, define

$$
\mu(A):=\sum \lambda_{n}(A) e_{n}
$$

Then $\mu$ is a vector measure in bva $\left.\mathscr{F}, c_{0}\right)$ which does not possess a Jordan decomposition in $b v a\left(\mathscr{F}, c_{0}\right)$. However, if $q: c_{0} \rightarrow l^{\infty}$ denotes the evaluation map, then the vector measure $q \circ \mu \in b v a\left(\mathscr{F}, l^{\infty}\right)$ has a Jordan decomposition in $b v a\left(\mathscr{F}, l^{\infty}\right)$ since $l^{\infty}$ is an order complete $A M$-space with unit and therefore has property $(P)$.

More generally, in order complete $A M$-spaces with unit, the Jordan decomposition theorem can be extended to the class of all bounded vector measures:

Theorem 5.2. Suppose $\mathbb{E}$ is an order complete $A M$-space with unit. Then the class $b a(\mathscr{F}, \mathbb{E})$ is an order complete Banach lattice for the norm $\|\cdot\| \cdot \|(\Omega)$, and the map $\mathscr{X}$ is an isometric vector lattice isomorphism of ba $(\mathscr{F}, \mathbb{E})$ onto $\mathscr{L}(\mathbb{D}, \mathbb{E})$. Moreover, the Banach lattices $o b a(\mathscr{F}, \mathbb{E})$ and $b a(\mathscr{F}, \mathbb{E})$ are identical.

Proof. Since $\mathbb{E}$ is an order complete $A M$-space with unit, we have $\mathscr{L}^{r}(\mathbb{D}, \mathbb{E})=$ $\mathscr{L}(\mathbb{D}, \mathbb{E})$, by $[10 ;$ Theorem IV.1.5]. Now the assertion follows from Theorem 3.1 and Theorem 4.1 .

An elementary proof of the Jordan decomposition theorem contained in the previous result was given in [11].

In order to complete the discussion of the Jordan decomposition for vector measures in an $A M$-space, we include two further results.

A vector measure $\mu \in a(\mathscr{F}, \mathbb{E})$ has relatively compact range if the set $\{\mu(A) \mid A \in \mathscr{F}\}$ is relatively compact. Let $c p a(\mathscr{F}, \mathbb{E})$ denote the class of all vector measures of relatively compact range-in $a(\mathscr{F}, \mathbb{E})$.

Let $\mathscr{K}(\mathbb{D}, \mathbb{E})$ denote the Banach space of all compact operators $\mathbb{D} \rightarrow \mathbb{E}$, endowed with the operator norm.

Theorem 5.3. Suppose $\mathbb{E}$ is an AM-space. Then the class cpa(F, $\mathbb{E})$ is a Banach lattice for the norm $\|\cdot\| \cdot \|(\Omega)$, and the restriction of $\mathscr{X}$ is an isometric vector lattice isomorphism of $\operatorname{cpa}(\mathscr{F}, \mathbb{E})$ onto $\mathscr{K}(\mathbb{D}, \mathbb{E})$.

Proof. It is not hard to verify that a bounded vector measure has relatively compact range if and only if its representing linear operator is compact. Since $\mathbb{E}$ is an $A M$-space, the class $\mathscr{K}(\mathbb{D}, \mathbb{E})$, endowed with the canonical order and the operator norm, is a Banach lattice; see [10; Theorem IV.4.6 Corollary 2].

Corollary 5.4. The Banach lattices $c p a\left(\mathscr{F}, c_{0}\right)$ and oba $\left(\mathscr{F}, c_{0}\right)$ are identical.

Proof. By Theorem 5.3, we have $c p a\left(\mathscr{F}, c_{0}\right) \subseteq o b a\left(\mathscr{F}, c_{0}\right)$. Since the order intervals in $c_{0}$ are relatively compact, we also have $o b a\left(\mathscr{F}, c_{0}\right) \subseteq c p a\left(\mathscr{F}, c_{0}\right)$. Also by Theorem 5.3, 
the norm $\|\cdot\|(\Omega)$ is a lattice norm on $c p a\left(\mathscr{F}, c_{0}\right)$, which yields

$$
\|\mu\|(\Omega)=\|\||\mu|\|(\Omega)=\||\mu|(\Omega) \|,
$$

for all $\mu \in \operatorname{cpa}\left(\mathscr{F}, c_{0}\right)=o b a\left(\mathscr{F}, c_{0}\right)$.

Corollary 5.4 can be used to establish the existence of a Jordan decomposition for countably additive vector measures in $c_{0}$ which are defined on the $\sigma$-algebra consisting of all subsets of $\Omega$; for the details, see Diestel and Faires [3] and Faires and Morrison [5]. A result which is closely related to Corollary 5.4 was also given by Niculescu [7].

In a Banach lattice $\mathbb{E}$ having property $(P)$, the projections associated with the YosidaHewitt decomposition of $b v a(\mathscr{F}, \mathbb{E})$ are orthogonal (with respect to the order of $\mathbb{E}$ ) and they are unique-nearest-point maps (with respect to the variation norm $\|\cdot\|(\Omega)$ ). In fact, if $\mu=\mu_{1}+\mu_{2}$ is the Yosida-Hewitt decomposition of $\mu \in b v a(\mathscr{F}, \mathbb{E})$, with $\mu_{1} \in \operatorname{bvca}(\mathscr{F}, \mathbb{E})$ and $\mu_{2} \in \operatorname{bvpfa}(\mathscr{F}, \mathbb{E})$, then we have $\left|\mu_{1}\right| \wedge\left|\mu_{2}\right|=0$. Moreover, for all $\phi \in b v c a(\mathscr{F}, \mathbb{E})$, the Yosida-Hewitt decomposition of $\mu-\phi$ is given by $\mu-\phi=\left(\mu_{1}-\phi\right)+\mu_{2}$, which yields

$$
\left\|\mu-\mu_{1}\right\|(\Omega)=\left\|\mu_{2}\right\|(\Omega) \leqq\left\|\mu_{1}-\phi\right\|(\Omega)+\left\|\mu_{2}\right\|(\Omega)=\left\|\mu_{1}-\phi+\mu_{2}\right\|(\Omega)=\|\mu-\phi\|(\Omega)
$$

by Theorem 4.8. Thus we have $\left\|\mu-\mu_{1}\right\|(\Omega)=\inf _{\text {buca F, E) }}\|\mu-\phi\|(\Omega)$. The previous inequality also reveals that $\phi=\mu_{1}$ holds for all $\phi \in \operatorname{bvca}(\mathscr{F}, \mathbb{E})$ satisfying $\|\mu-\phi\|(\Omega)=$ $\left\|\mu-\mu_{1}\right\|(\Omega)$. Therefore, the projection $b v a(\mathscr{F}, \mathbb{E}) \rightarrow b v c a(\mathscr{F}, \mathbb{E})$ is a unique-nearest-point map, and the same is true for the projection $b v a(\mathscr{F}, \mathbb{E}) \rightarrow b v p f a(\mathscr{F}, \mathbb{E})$.

In the case where $\mathbb{E}$ is a Banach space, the relationship between the Yosida-Hewitt decomposition and some type of orthogonality and optimal approximation was also studied by Bilyeu and Lewis [1]. Let $\operatorname{cpca}(\mathscr{F}, \mathbb{E})$ denote the class of all countably additive vector measures in $c p a(\mathscr{F}, \mathbb{E})$. A vector measure $\mu \in c p a(\mathscr{F}, \mathbb{E})$ is purely finitely additive if $e^{\prime} \circ \mu \in b v p f a(\mathscr{F}, \mathbb{R})$ holds for each $e^{\prime} \in \mathbb{E}^{\prime}$; this definition is in accordance with the definition given in Section 3. By the Yosida-Hewitt decomposition theorem of Uhl [14], each vector measure in $c p a(\mathscr{F}, \mathbb{E})$ is the sum of a vector measure in $c p c a(\mathscr{F}, \mathbb{E})$ and a purely finitely additive vector measure in $c p a(\mathscr{F}, \mathbb{E})$, and the decomposition is unique. Recall that a vector $x \in \mathbb{E}$ is James orthogonal to $y \in \mathbb{E}$ if $\|x\|=\inf _{\mathbf{R}}\|x+\alpha y\|$, and that a Banach space $\mathbb{H}$ is smooth if, for each $h \in \mathbb{H}$ satisfying $\|h\|=1$, there exists a unique $h^{\prime} \in \mathbb{H}^{\prime}$ satisfying $\left\|h^{\prime}\right\|=h^{\prime}(h)=1$. Bilyeu and Lewis [1] proved the following result:

Theorem 5.5. Suppose $\mathbb{E}$ has a smooth dual. Then a vector measure in cpa $(\mathscr{F}, \mathbb{E})$ is purely finitely additive if and only if it is James orthogonal to each vector measure in $\operatorname{cpca}(\mathscr{F}, \mathbb{E})$, and the projection $c p a(\mathscr{F}, \mathbb{E}) \rightarrow c p c a(\mathscr{F}, \mathbb{E})$ associated with the Yosida-Hewitt decomposition of cpa $(\mathscr{F}, \mathbb{E})$ is a unique-nearest-point map with respect to the semivariation norm $\|\cdot \cdot\|(\Omega)$.

It appears, however, that in this result the role of purely finitely additive vector 
measures and of countably additive vector measures cannot be interchanged since the relation of James orthogonality fails to be symmetric.

We finally remark that the Lebesgue null-set decomposition theorem remains valid if the vector measure $\phi$ is assumed to take its values in a different Riesz space $\mathbb{R}-$; in particular, $\phi$ may be assumed to be real-valued. Similarly, the Lebesgue decomposition theorem (of Rickart and Uhl) remains valid if the real-valued set function $\lambda$ is replaced by a vector measure $\phi$ which takes its values in some Banach space $\mathbb{H}$ and if $\lambda$-continuous and $\lambda$-singular vector measures are replaced by $\|\phi\|$-continuous and $\|\phi\|$-singular vector measures.

\section{REFERENCES}

1. R. C. BILYeU and P. W. Lewis, Orthogonality and the Hewitt-Yosida theorem in spaces of measures, Rocky Mountain J. Math. 7 (1977), 629-638.

2. M. Congost Iglesias, Medidas y probabilidades en estructuras ordenadas, Stochastica 5 (1981), 45-68.

3. J. Diestel and B. Faires, On vector measures, Trans. Amer. Math. Soc. 198 (1974), 253-271.

4. J. Diestel and J. J. Uhl JR., Vector Measures (Providence, Rhode Island: Amer. Math. Soc. 1977).

5. B. Faires and T. J. Morrison, The Jordan decomposition of vector-valued measures, Proc. Amer. Math. Soc. 60 (1976), 139-143. 1971).

6. W. A. J. Luxemburg and A. C. ZaAnen, Riesz Spaces I (Amsterdam-London: North-Holland,

7. C. P. Niculescu, Jordan decomposition and locally absolutely continuous operators, Revue Roumaine Math. Pures Appl. 21 (1976), 343-352.

8. P. K. Pavlakos, The Lebesgue decomposition theorem for partially ordered semigroupvalued measures, Proc. Amer. Math. Soc. 71 (1978), 207-211.

9. C. E. RicKart, Decomposition of additive set functions, Duke Math. J. 10 (1943), 653-665.

10. H. H. Schaefer, Banach Lattices and Positive Operators (Berlin-Heidelberg-New York: Springer, 1974).

11. K. D. Schmid, On the Jordan decomposition for vector measures, Probability in Banach Spaces IV (Lecture Notes in Mathematics, vol. 990, Berlin-Heidelberg-New York: Springer, 1983), 198-203.

12. K. D. Schmidt, Amarts-a measure theoretic approach, Amarts and Set Function Processes (Lecture Notes in Mathematics, vol. 1042, Berlin-Heidelberg-New York: Springer, 1983), 51-236.

13. M. TALAGRAND, Quand l'espace des mesures à variation bornée est-il faiblement séquentiellement complet? Proc. Amer. Math. Soc. 90 (1984), 285-288.

14. J. J. UHL JR., Extensions and decompositions of vector measures, J. London Math. Soc. (2) 3 (1971), 672-676.

Seminar fór Statistik, Universität Mannheim, A5

6800 Mannheim, West Germany 